\section{Obesidad y cáncer en Chile: estimación de las fracciones atribuibles poblacionales}

\author{
MARÍA LUISA GARMENDIA ${ }^{1}$, PABLO RUIZ ${ }^{2, a}$, RICARDO UAUY ${ }^{1}$
}

\section{Obesity and cancer in Chile: Estimation of population attributable fractions}

Background: Obesity is a risk factor for the development of certain types of cancer. Aim: To estimate the proportion of cancers potentially attributable to obesity in men and women in Chile based on the calculation of population attributable fractions (PAF \%). Material and Methods: Cancer sites studied were those where obesity is a known risk factor based on the updated World Cancer Research Fund (WCRF) analysis. Namely, colorectal, endometrium, esophagus, breast, pancreas, kidney and gallbladder cancers were analyzed. Overall and specific PAFs\% were calculated for cancer sites and sex from known estimates of relative risk and national prevalence of overweight and obesity. Results: The overall estimates of cancer PAF\% for obesity were approximately 20\%, without differences between men and women. Highest cancer PAFs\% were for endometrial (47\%) in women, and esophageal (35\%) and pancreatic (31\%) in men. The largest sex differences in PAFs $\%$ were for gallbladder (higher in women) and colorectal (higher in men). Results are closer to those reported from developed countries (USA and United Kingdom) than those from developing countries (Brasil, China). Conclusions: In Chile about 20\% of all cancers could be prevented by obesity prevention and control strategies.

(Rev Med Chile 2013; 141: 987-994).

Key words: Chile; Neoplasms; Public health; Obesity.

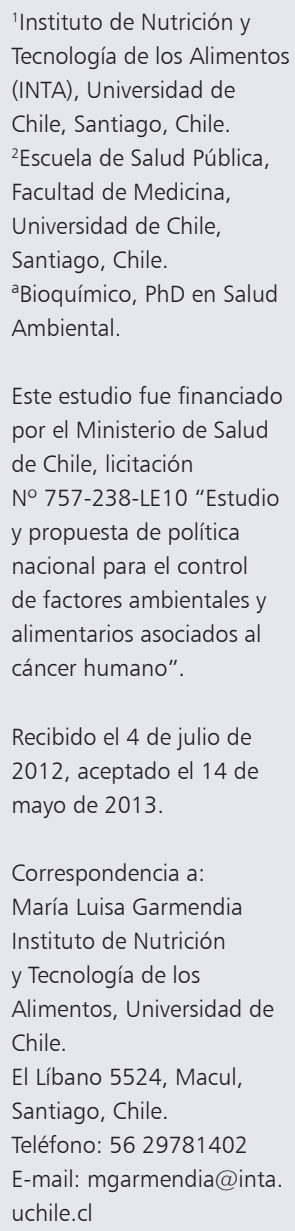

Este estudio fue financiado por el Ministerio de Salud de Chile, licitación No 757-238-LE10 "Estudio y propuesta de política nacional para el control de factores ambientales $y$ alimentarios asociados al cáncer humano".

Recibido el 4 de julio de 2012, aceptado el 14 de mayo de 2013.

Correspondencia a: María Luisa Garmendia Instituto de Nutrición y Tecnología de los Alimentos, Universidad de Chile.

El Líbano 5524, Macul, Santiago, Chile. Teléfono: 5629781402 E-mail:mgarmendia@inta. uchile.cl

$\mathrm{E}$ 1 cáncer constituye la segunda causa de muerte a nivel mundial y nacional, con más de 7 millones de muertes registradas a nivel mundial el año 2008 ${ }^{1}$; en Chile la tasa de mortalidad para el año 2009 fue de 133 muertes por cáncer cada cien mil habitantes ${ }^{2}$. En relación a la incidencia, en el año 2008 ocurrieron en el mundo 12 millones de casos nuevos de cáncer (excluyendo los cánceres de piel), mientras en Chile, la Agencia Internacional para la Investigación del Cáncer (IARC) estima tasas de incidencia semejantes a las de países desarrollados ${ }^{3}$.

Es importante conocer las causas del cáncer de modo de identificar medidas de prevención. Se estima que una fracción muy pequeña de los cánceres (5 a 10\%) está asociada a factores genéticos mientras el resto se atribuye a la exposición de la población a factores externos tales como tabaquismo, nutrición, agentes infecciosos, medicamentos, radiación ionizante y agentes químicos ${ }^{1,4}$. Por lo tanto, el cáncer se considera una enfermedad potencialmente evitable.

Es materia principal de la salud pública intentar determinar la fracción de los casos de cáncer atribuible a la exposición a los factores de riesgo conocidos, de modo de identificar las medidas de prevención más costo-efectivas. El cálculo de la fracción atribuible poblacional (FAP\%) permite estimar el grado en que los casos de cáncer son prevenibles, al disminuir o eliminar la exposición a un determinado factor de riesgo ${ }^{5-7}$. La FAP\% nos informa del porcentaje de casos que pueden 
evitarse al eliminar la exposición al factor de riesgo $^{8}$. Así, una $\mathrm{FAP} \%$ de $50 \%$ para la exposición al factor de riesgo $\mathrm{X}$ indica que al eliminar dicha exposición se espera que disminuyan en $50 \%$ los casos de cáncer.

El uso de FAP\% se ha generalizado por su fácil interpretación y relativa facilidad en su cálculo: este se basa en estimados de fuerza de asociación (riesgo relativo) y prevalencias de exposición. Aunque es necesario que los estimados de prevalencia de exposición al factor de riesgo sean de origen local, para los estimados de riesgo relativo es usual usar valores de referencia encontrados en la literatura internacional, en caso de no contar con estudios locales. Factores de riesgo con una FAP\% alta, esto es, con un potencial alto de prevención en caso de cesar la exposición, sólo se observan si tanto la prevalencia de exposición como el grado de asociación son altos. A nivel mundial, los factores de riesgo con $\mathrm{FAP} \%$ más altas para cáncer son el tabaco y la nutrición (incluyendo la dieta no saludable, la inactividad física y el exceso de peso), estimándose que alrededor de un tercio de los casos de cáncer se pueden prevenir eliminando la exposición al tabaco, y otro tercio mejorando la nutrición, lo que prevendría 3-4 millones de casos de cáncer al año a nivel mundial ${ }^{9}$. Entre los factores nutricionales, la obesidad, tanto general como abdominal, es sin duda el factor que ha demostrado mayor evidencia causal con cánceres de varias localizaciones: colorectal, endometrio, esófago, mama, páncreas, riñón y vesícula biliar ${ }^{9}$.

Chile ha experimentado una transición epidemiológica rápida en las últimas décadas, encontrándose altas prevalencias de sobrepeso y obesidad ${ }^{10}$. Dado que existe evidencia convincente de la asociación entre obesidad y varias localizaciones de cáncer, el objetivo de este estudio fue estimar la proporción de cánceres que pudieran ser potencialmente prevenibles en Chile a través del control de la obesidad, mediante el cálculo de FAP\%, y compararla con estimaciones similares realizadas en otros países.

\section{Material y Método}

\section{Diseño y Población}

Se realizó una estimación del riesgo de cáncer atribuible a la obesidad en Chile utilizando la metodología de FAP\%; incluyó aquellas localizaciones en que la obesidad es un factor de riesgo conocido de cáncer. A partir de los cálculos de FAP\% por localización específica y sexo, se calcularon FAP\% globales, esto es, el impacto de la obesidad en las tasas globales de incidencia de cáncer en Chile.

\section{Cálculo de las fracciones atribuibles poblacionales (FAP\%)}

Se calcularon las FAP\% para Chile replicando la misma metodología utilizada anteriormente en 4 países (Brasil, Estados Unidos, Reino Unido y China $)^{9}$. En esta metodología, la FAP\% se calculó asumiendo 3 categorías de exposición -alta, media y baja- y estimando riesgos relativos para las tres categorías, dejando la categoría baja como referencia. La Ecuación 1 muestra este cálculo:

\section{Ecuación 1}

$$
F A P \%=\frac{\left[P_{1} x\left(R R_{1}-1\right)\right]+\left[P_{2} x\left(R R_{2}-1\right)\right]+\left[P_{3} x\left(R R_{3}-1\right)\right]}{1+\left[P_{1} x\left(R R_{1}-1\right)\right]+\left[P_{2} x\left(R R_{2}-1\right)\right]+\left[P_{3} x\left(R R_{3}-1\right)\right]}
$$

Donde $P_{1}$ y $R R_{1}$ son la prevalencia y riesgo relativo para la categoría de baja exposición, mientras que $P_{2}, P_{3}, R R_{2}$ y $R R_{3}$ son las prevalencias y riesgos relativos para las categorías de exposición media y alta, respectivamente. Por ser el grupo de referencia, a $R R_{1}$ se le asigna un valor de 1 . Los rangos de confianza de cada FAP\% se calculan a partir de los límites de confianza de los RR.

Se calculó un estimado global de FAP\% en base a las FAP\% por localización específica, ponderando por las tasas de incidencia de cada localización según la Ecuación 2.

Ecuación 2

$$
F A P \%(\text { global })=1-\prod_{i}\left(1-\frac{I_{i}}{I} F A P \%{ }_{i}\right)
$$

Donde FAP\% es la FAP\% para la localización i y $I / I$ es la proporción de casos incidentes de cáncer para la localización i con respecto al total de las 7 localizaciones en estudio.

\section{Tasas de incidencia y mortalidad por cáncer}

Se obtuvieron las tasas de mortalidad y de incidencia por 100.000 habitantes para el año 2008 a partir del GLOBOCAN (ajustadas por edad según la población estándar mundial) ${ }^{3}$ En el caso de Chile, las incidencias publicadas son estimaciones, 
dado que no existen registros nacionales de cáncer, sólo regionales.

\section{Riesgos relativos}

Para las siete localizaciones de cáncer (colorrectal, endometrio, esófago, mama, páncreas, riñón y vesícula biliar) en las cuales la obesidad (medida a través del índice de masa corporal (IMC) $(\mathrm{kg} /$ $\left.\mathrm{m}^{2}\right)$ ), es un factor de riesgo ${ }^{9}$, los estimados de riesgo relativo para cada localización se basaron en un estudio de cohorte, cuyo tamaño de efecto fuera representativo de todos los estudios existentes y que además tuviera puntos de corte que permitieran comparar categorías de exposición alta, media y baja. Los estimados de riesgo relativo por categoría de exposición y sexo se muestran en la Tabla 1 junto con los estudios de referencia. La mayor fuerza de asociación entre obesidad y cáncer se observó para los cánceres de endometrio en las mujeres y esófago para ambos sexos.

\section{Prevalencias de obesidad}

Para comparar las prevalencias de obesidad entre Chile y otros países se usaron los estimados del año 2008 de la Organización Mundial de la Salud para personas de 20 años de edad o más ${ }^{11}$. Para el cálculo de FAP\% para Chile, se estimaron las prevalencias de obesidad para hombres y mujeres utilizando la base de datos de la Encuesta Nacional de Salud del año 2010, a las que se aplicó los factores de expansión correspondientes. Para el cálculo de las prevalencias para cada categoría de exposición, los puntos de corte del IMC variaron según la localización del cáncer, de acuerdo a los estudios de referencia de los que se obtuvieron los riesgos relativos. Así para las localizaciones colorrectal, endometrio, esófago, riñón y vesícula biliar, las categorías alta, media y baja fueron $\geq 30, \geq 25$ y $<29,9$ y $<25 \mathrm{~kg} /$ $\mathrm{m}^{2}$ de IMC, respectivamente; mientras que para mama fueron $\geq 25, \geq 21 \mathrm{y}<24,9 \mathrm{y}<21 \mathrm{~kg} / \mathrm{m}^{2}$ y para páncreas fueron $\geq 30, \geq 23 \mathrm{y}<29,9 \mathrm{y}<23 \mathrm{~kg} / \mathrm{m}^{2}$.

Tabla 1. Riesgos relativos de cáncer y obesidad según localización y sexo

\begin{tabular}{|c|c|c|c|}
\hline \multirow[b]{2}{*}{ Localización } & \multirow[b]{2}{*}{ Autor, año } & \multicolumn{2}{|c|}{ RR (IC 95\%) } \\
\hline & & Alta vs baja exposición & Media vs baja exposición \\
\hline Colorrectal (C18-C20) & Engeland, $2005^{27}$ & & \\
\hline Hombres & & $1,4(1,3-1,5)$ & $1,2(1,1-1,2)$ \\
\hline Mujeres & & $1,1(1,0-1,1)$ & $1,0(1,0-1,1)$ \\
\hline Endometrio (C54) & Silvera, $2005^{28}$ & & \\
\hline Hombres & & - & - \\
\hline Mujeres & & $3,4(2,7-4,3)$ & $1,4(1,1-1,8)$ \\
\hline Esófago (C15) & Lindblad, $2005^{29}$ & & \\
\hline Hombres & & $1,8(1,0-3,0)$ & $1,9(1,3-2,8)$ \\
\hline Mujeres & & $2,1(1,0-4,7)$ & $1,1(0,5-2,3)$ \\
\hline Mama (C50) & Van der Brandt, $2000^{30}$ & & \\
\hline Hombres & & - & - \\
\hline Mujeres & & $1,3(1,1-1,5)$ & $1,1(1,0-1,3)$ \\
\hline Páncreas (C25) & Michaud, $2000^{31}$ & & \\
\hline Hombres & & $1,8(0,9-3,5)$ & $1,5(0,9-2,5)$ \\
\hline Mujeres & & $1,7(1,1-2,6)$ & $1,3(0,9-1,8)$ \\
\hline Riñón (C64-C66, C68) & Bjorge, $2004^{32}$ & & \\
\hline Hombres & & $1,6(1,4-1,8)$ & $1,2(1,1-1,3)$ \\
\hline Mujeres & & $1,9(1,7-2,1)$ & $1,3(1,2-1,5)$ \\
\hline Vesícula biliar (C23) & Engeland, $2005^{27}$ & & \\
\hline Hombres & & $1,4(1,0-1,9)$ & $1,0(0,8-1,2)$ \\
\hline Mujeres & & $1,9(1,6-2,2)$ & $1,3(1,1-1,5)$ \\
\hline
\end{tabular}

Adaptado de Informe "Policy and Action for Cancer Prevention", World Cancer Research Fund/American Institute for Cancer Research (WCRF/AICR). RR: riesgo relativo. IC: intervalo de confianza. 
Tabla 2. Tasas de incidencia y mortalidad para cáncer año 2008, según país, localización y sexo

\begin{tabular}{|c|c|c|c|c|c|c|c|c|c|c|c|c|}
\hline \multirow[t]{2}{*}{ Localización } & \multicolumn{2}{|c|}{ Chile } & \multicolumn{2}{|c|}{$\begin{array}{l}\text { Latino- } \\
\text { américa }\end{array}$} & \multicolumn{2}{|c|}{ Brasil } & \multicolumn{2}{|c|}{$\begin{array}{l}\text { Estados } \\
\text { Unidos }\end{array}$} & \multicolumn{2}{|c|}{$\begin{array}{l}\text { Reino } \\
\text { Unido }\end{array}$} & \multicolumn{2}{|c|}{ China } \\
\hline & Inc. ${ }^{a}$ & Mort. ${ }^{\text {b }}$ & Inc. ${ }^{a}$ & Mort. ${ }^{b}$ & Inc. ${ }^{a}$ & Mort. ${ }^{b}$ & Inc. ${ }^{a}$ & Mort. ${ }^{b}$ & Inc. ${ }^{a}$ & Mort. ${ }^{b}$ & Inc. ${ }^{a}$ & Mort. ${ }^{b}$ \\
\hline \multicolumn{13}{|c|}{ Colorrectal (C18-C20) } \\
\hline Hombres & 14,4 & 8,8 & 12,1 & 7,1 & 12,0 & 6,5 & 34,1 & 9,9 & 37,3 & 13,9 & 16,3 & 8,0 \\
\hline Mujeres & 11,9 & 6,9 & 10,9 & 6,1 & 11,4 & 5,8 & 25 & 7,7 & 25,3 & 9,1 & 12,2 & 5,9 \\
\hline \multicolumn{13}{|c|}{ Endometrio (C54) } \\
\hline \multicolumn{13}{|l|}{ Hombres } \\
\hline Mujeres & $\underline{2,3}$ & 1,0 & 5,1 & 2,0 & 4,5 & 2,0 & 16,5 & 2,5 & 13,2 & 2,0 & 11,1 & 2,4 \\
\hline \multicolumn{13}{|l|}{ Esófago (C15) } \\
\hline Hombres & 5,4 & 4,8 & 5,2 & 4,6 & 8,2 & 7,3 & 5,8 & 4,9 & 9,5 & 8,6 & 22,9 & 16,7 \\
\hline Mujeres & 3,3 & 2,5 & 1,7 & 1,3 & 2,5 & 1,8 & 1,2 & 1,0 & 3,6 & 3,1 & 10,5 & 8,2 \\
\hline \multicolumn{13}{|l|}{ Mama (C50) } \\
\hline \multicolumn{13}{|l|}{ Hombres } \\
\hline Mujeres & 40,1 & 11,0 & 39,7 & 12,4 & 42,3 & 12,3 & 76,0 & 14,7 & 89,1 & 18,6 & 21,6 & 5,7 \\
\hline \multicolumn{13}{|l|}{ Páncreas (C25) } \\
\hline Hombres & 5,3 & 5,1 & 4,6 & 4,4 & 4,3 & 4,3 & 8,0 & 7,3 & 6,8 & 6,5 & 3,2 & 2,9 \\
\hline Mujeres & 5,4 & 5,1 & 3,9 & 3,8 & 3,4 & 3,3 & 6,1 & 5,1 & 5,4 & 5,2 & 2,4 & $\underline{2,2}$ \\
\hline \multicolumn{13}{|c|}{ Riñón (C64-C66, C68) } \\
\hline Hombres & 7,8 & 4,5 & 4,1 & 2,3 & 6,1 & 2,6 & 16,1 & 3,6 & 10,4 & 4,2 & 2,8 & 0,9 \\
\hline Mujeres & 3,7 & 2,1 & 2,2 & 1,1 & 1,6 & 0,9 & 8,7 & 1,7 & 5,3 & 2,1 & 1,5 & $\underline{0,5}$ \\
\hline \multicolumn{13}{|c|}{ Vesícula biliar (C23) } \\
\hline Hombres & 7,0 & 5,8 & 2,1 & 1,6 & 1,4 & 1,3 & 1,9 & 0,5 & 0,3 & 0,3 & 1,5 & 1,1 \\
\hline Mujeres & 13,4 & 11,5 & 3,7 & 3,0 & 2,4 & 2,3 & 1,6 & 0,6 & 0,7 & 0,5 & 1,8 & 1,3 \\
\hline
\end{tabular}

(a) Inc: Tasas de incidencia; casos de cáncer anuales por cada 100.000 habitantes ajustadas por edad. (b) Mort.: Tasas de mortalidad: muertes anuales por cada 100.000 habitantes ajustadas por edad. Negrita: tasa más alta de la fila. Subrayado: tasa más baja de la fila. Fuente: GLOBOCAN, Agencia Internacional para la Investigación del Cáncer (IARC).

\section{Resultados}

\section{Distribución de cáncer en Chile}

La Tabla 2 muestra las tasas de incidencia y mortalidad ajustadas por edad por cada 100 mil habitantes para los principales cánceres asociados a obesidad en Chile y en otros países. En Chile, las 7 localizaciones de cáncer asociadas a obesidad representan en su conjunto $21 \%$ de las muertes en hombres y $30 \%$ de las muertes en mujeres. En general, Chile muestra tasas de incidencia y mortalidad parecidas a los países latinoamericanos, en contraste con países como Estados Unidos de Norteamérica y Reino Unido que muestran tasas mucho más altas para colorrectal, endometrio, mama y riñón. Destacamos que Chile presenta una de las tasas más altas a nivel mundial para cáncer de vesícula biliar.

\section{Prevalencia de obesidad}

La Tabla 3 muestra las prevalencias de obesidad en población adulta (igual o mayor a 20 años) para Chile y otros países ${ }^{11}$. En el contexto sudamericano, Chile es el segundo país con más obesidad en mujeres $(33,6 \%)$, después de Venezuela; y tercero en hombres $(24,5 \%)$ luego de Argentina y Venezuela. A nivel global, Chile presenta prevalencias cercanas o mayores incluso que países desarrollados como Estados Unidos de Norteamérica y Reino Unido; y mucho más altas que otros países emergentes como China.

\section{Fracciones atribuibles poblacionales (FAP\%)}

En La Tabla 4 se muestran los estimados de FAP\% por sexo y localización y la comparación con otros países. Las FAP\% más altas para Chile (>20\%) fueron las de endometrio, esófago, vesí- 
Tabla 3. Prevalencia de obesidada año 2008, según país y sexo

\begin{tabular}{|lcc|}
\hline País & Hombres (\%) & Mujeres (\%) \\
\hline Chile & 24,5 & 33,6 \\
\hline Argentina & 27,4 & 31,0 \\
\hline Perú & 11,1 & 21,7 \\
\hline Bolivia & 10,0 & 27,1 \\
\hline Paraguay & 16,2 & 22,3 \\
\hline Colombia & 11,9 & 23,7 \\
\hline Venezuela & 26,6 & $\mathbf{3 4 , 4}$ \\
\hline Uruguay & 20,7 & 26,0 \\
\hline Ecuador & 15,7 & 28,2 \\
\hline Brasil & 16,5 & 22,1 \\
\hline Estados Unidos & $\mathbf{3 0 , 2}$ & 33,2 \\
\hline Reino Unido & 24,4 & 25,2 \\
\hline China & $\underline{4,6}$ & $\underline{6,5}$ \\
\hline
\end{tabular}

(a) Definida como índice de masa corporal $\geq 30 \mathrm{~kg} / \mathrm{m}^{2}$ en personas de 20 años de edad o más. Negrita: prevalencia más alta de la columna Subrayado: prevalencia más baja de la columna. Fuente: Estadísticas Sanitarias Mundiales, 2012. Organización Mundial de la Salud.

cula biliar, riñón y páncreas en mujeres; y esófago y páncreas en hombres. Las mayores diferencias según sexo se encontraron para vesícula biliar (mayor en mujeres) y colorrectal (mayor en hombres). El estimado global de FAP\% fue cercano al 20\% tanto en mujeres como en hombres.

En relación a los países con los que se hizo la comparación, las FAP\% más elevadas se encuentran en Estados Unidos de Norteamérica y en segundo lugar en el Reino Unido, mientras que las más bajas se encuentran en China y Brasil. Los resultados de Chile se acercan mucho más a las FAP\% estimadas en Estados Unidos y Reino Unido que a las de Brasil y China.

\section{Discusión}

El presente estudio estimó las FAP\% para cáncer en Chile debido a obesidad a nivel global y para distintas localizaciones. Se encontraron FAP\% altas para endometrio, esófago, riñón y vesícula biliar en mujeres; y en esófago y páncreas en hombres. A nivel global se estimó que cerca de $20 \%$ de los cánceres en Chile se atribuyen a obesidad tanto para hombres como para mujeres. Esto quiere decir que una fracción importante (20\% o más) de los cánceres tanto en algunas localizaciones específicas, como a nivel global, podrían prevenirse si se disminuyen los niveles de obesidad del país.

Las FAP\% estimadas para Chile se acercan mucho más a las FAP\% estimadas para Estados Unidos y Reino Unido que a las de Brasil y China, lo que podría indicar que la rápida transición nutricional que ha experimentado Chile en las últimas décadas, reflejada en cambios acelerados en estilos de vida, especialmente dieta y actividad física, ha llevado a que en la actualidad las prevalencias de obesidad sean más cercanas a las de los países industrializados ${ }^{12,13}$.

La metodología utilizada para el análisis de este estudio presenta varias limitaciones ${ }^{14}$. Primero, el riesgo se estableció de acuerdo a tres categorías de exposición (alta, media y baja), cuando es posible que en realidad exista un gradiente continuo de riesgo. En segundo lugar, los RR para el cálculo de las FAP\% se basaron en la extrapolación del riesgo a partir de un estudio internacional que podría no reflejar el riesgo asociado a cáncer por obesidad en la población chilena ${ }^{15}$. Tercero, las cohortes que han evaluado la relación entre cáncer y obesidad se han centrado en personas de edad media, lo que probablemente no refleja los riesgos a edades jóvenes o envejecidas ${ }^{16}$. Cuarto, los estimados de FAP\% para cáncer de mama debieran ser calculados diferencialmente en mujeres premenopáusicas y postmenopáusicas, dado que la evidencia epidemiológica señala que la obesidad estaría asociada a un mayor riesgo de este cáncer sólo en postmenopáusicas ${ }^{17}$. Finalmente, los datos actuales de incidencia representan el impacto por exposición a obesidad en décadas pasadas, donde las prevalencias eran más bajas que las actuales ${ }^{18}$.

Estos resultados tienen implicancias para las políticas públicas de nuestro país. Dadas las magnitudes de FAP\% observadas, estrategias que apunten a reducir la obesidad pronostican una reducción importante en la incidencia de cáncer. Dado que otros factores de riesgo nutricionales, tales como el sedentarismo y el alto consumo de sal, también tienen un impacto en cáncer, una intervención nutricional integral podría tener un efecto amplificador en la reducción de la incidencia de cáncer a nivel poblacional. Además, todos estos factores tienen impactos conocidos en otras 
Tabla 4. Fracciones atribuibles poblacionales (FAP\%) de cáncer por obesidad en varios países según localización y sexo

\begin{tabular}{|c|c|c|c|c|c|c|c|c|c|c|}
\hline Localización cáncer & \multicolumn{2}{|r|}{ Chile } & \multicolumn{2}{|r|}{ Brasil } & \multicolumn{2}{|c|}{$\begin{array}{l}\text { FAP\% (Rango) } \\
\text { Estados Unidos }\end{array}$} & \multicolumn{2}{|c|}{ Reino Unido } & \multicolumn{2}{|c|}{ China } \\
\hline $\begin{array}{l}\text { Colorrectal }(\mathrm{C} 18-\mathrm{C} 20) \\
\text { Hombres } \\
\text { Mujeres }\end{array}$ & $\begin{array}{r}13 \\
3\end{array}$ & $\begin{array}{l}(10-15) \\
(\mathbf{0}, \mathbf{3}-\mathbf{5})\end{array}$ & $\begin{array}{l}8 \\
1\end{array}$ & $\begin{array}{l}(6-9) \\
(0-3) \\
\end{array}$ & $\begin{array}{r}16 \\
3\end{array}$ & $\begin{array}{c}(13-16) \\
(0-5)\end{array}$ & $\begin{array}{r}14 \\
2\end{array}$ & $\begin{array}{c}(11-16) \\
(0-3)\end{array}$ & $\frac{5}{1}$ & $\frac{(4-6)}{(0-2)}$ \\
\hline $\begin{array}{l}\text { Endometrio (C54) } \\
\text { Hombres } \\
\text { Mujeres }\end{array}$ & 47 & $(36-56)$ & 29 & $(20-39)$ & 49 & $(39-58)$ & 38 & $(27-48)$ & $\underline{18}$ & $(10-25)$ \\
\hline $\begin{array}{c}\text { Esófago (C15) } \\
\text { Hombres } \\
\text { Mujeres }\end{array}$ & $\begin{array}{l}35 \\
27\end{array}$ & $\begin{array}{c}(11-55) \\
(0-6)\end{array}$ & $\begin{array}{l}20 \\
26\end{array}$ & $\begin{array}{l}(5-39) \\
(0-54)\end{array}$ & $\begin{array}{l}32 \\
\mathbf{3 8}\end{array}$ & $\begin{array}{l}(6-55) \\
\mathbf{( 0 - 6 6 )}\end{array}$ & $\begin{array}{l}29 \\
33\end{array}$ & $\begin{array}{l}(6-52) \\
(0-61)\end{array}$ & $\frac{14}{20}$ & $\frac{(3-30)}{(0-44)}$ \\
\hline $\begin{array}{c}\text { Mama (C50) } \\
\text { Hombres } \\
\text { Mujeres }\end{array}$ & 17 & $(5-28)$ & 14 & $(4-22)$ & 17 & $(6-26)$ & 16 & $(5-25)$ & $\underline{12}$ & $(4-20)$ \\
\hline $\begin{array}{l}\text { Páncreas (C25) } \\
\text { Hombres } \\
\text { Mujeres }\end{array}$ & $\begin{array}{l}31 \\
\mathbf{2 6}\end{array}$ & $\begin{array}{l}(0-59) \\
\mathbf{( 0 - 4 7 )}\end{array}$ & $\begin{array}{l}25 \\
14\end{array}$ & $\begin{array}{l}(0-51) \\
(0-36)\end{array}$ & $\begin{array}{l}34 \\
25\end{array}$ & $\begin{array}{l}(\mathbf{0}-62) \\
(0-48)\end{array}$ & $\begin{array}{l}32 \\
19\end{array}$ & $\begin{array}{l}(0-60) \\
(0-42)\end{array}$ & $\frac{20}{10}$ & $\frac{(0-45)}{(0-30)}$ \\
\hline $\begin{array}{l}\text { Riñón (C64-C66, C68) } \\
\text { Hombres } \\
\text { Mujeres }\end{array}$ & $\begin{array}{l}16 \\
27\end{array}$ & $\begin{array}{l}(11-21) \\
(21-32)\end{array}$ & $\begin{array}{l}10 \\
16\end{array}$ & $\begin{array}{c}(6-13) \\
(12-20)\end{array}$ & $\begin{array}{l}20 \\
28\end{array}$ & $\begin{array}{l}(14-26) \\
(23-33)\end{array}$ & $\begin{array}{l}17 \\
21\end{array}$ & $\begin{array}{l}(12-23) \\
(17-26)\end{array}$ & $\underline{6}$ & $\frac{(4-9)}{(8-14)}$ \\
\hline $\begin{array}{l}\text { Vesícula biliar (C23) } \\
\text { Hombres } \\
\text { Mujeres }\end{array}$ & $\begin{array}{r}7 \\
27\end{array}$ & $\begin{array}{c}(0-20) \\
(18-35)\end{array}$ & $\begin{array}{r}3 \\
15\end{array}$ & $\begin{array}{l}(0-12) \\
(9-22)\end{array}$ & $\begin{array}{l}11 \\
28\end{array}$ & $\begin{array}{c}(0-27) \\
(19-36)\end{array}$ & $\begin{array}{r}8 \\
21\end{array}$ & $\begin{array}{c}(0-22) \\
(13-28)\end{array}$ & $\begin{array}{r}\underline{2} \\
10 \\
\end{array}$ & $\frac{(0-7)}{\underline{(5-14)}}$ \\
\hline $\begin{array}{l}\text { Global } \\
\text { Hombres } \\
\text { Mujeres }\end{array}$ & $\begin{array}{l}17 \\
18\end{array}$ & $\begin{array}{l}(7-25) \\
(7-22)\end{array}$ & $\begin{array}{l}13 \\
13\end{array}$ & $\begin{array}{l}(3-23) \\
(4-21)\end{array}$ & $\begin{array}{l}20 \\
19\end{array}$ & $\begin{array}{c}(10-28) \\
(9-28)\end{array}$ & $\begin{array}{l}18 \\
16\end{array}$ & $\begin{array}{l}(8-27) \\
(6-25)\end{array}$ & $\frac{11}{12}$ & $\frac{(2-22)}{(2-23)}$ \\
\hline
\end{tabular}

Negrita: FAP\% más alto de la fila. Subrayado: FAP\% más bajo de la fila.

enfermedades crónicas tales como enfermedades cardiovasculares, hipertensión y diabetes.

Políticas nutricionales integrales dirigidas al control de la obesidad se han implementado en Chile en la última década a través de diversos programas -programa EGO-Chile, Programa de Salud Cardiovascular- enfocándose en promoción de salud y estilos de vida saludable. Sin embargo, las cifras de sobrepeso y obesidad en escolares, embarazadas y adultos son altas y se encuentran en aumento, lo que indica que estos esfuerzos han sido insuficientes ${ }^{10,19}$. Las recomendaciones de los organismos internacionales para la prevención de cánceres asociados a la nutrición van dirigidas a la mantención del peso corporal dentro de límites normales (ojalá acercando al límite más bajo del rango normal de IMC), consumir una dieta saludable, realizar actividad física y promover la lactancia materna. Sin embargo, estas recomendaciones difícilmente podrán ser alcanzadas si no se acompañan de iniciativas estructurales que faciliten su cumplimiento ${ }^{20}$. Algunas medidas exitosas en este ámbito han sido la regulación de alimentos no saludables (publicidad, impuestos específicos), subvención de productos saludables, el rotulado de los alimentos, involucrar a la industria en mejoras de productos, entre otras ${ }^{21}$. Respecto a la actividad física, se deben fomentar estrategias dirigidas a facilitar que las personas puedan caminar y hacer uso de la bicicleta y del transporte público por sobre el privado ${ }^{22-25}$.

En conclusión, este es el primer estudio que estima la $\mathrm{FAP} \%$ para cáncer debido a obesidad en Chile. Los resultados pronostican un gran impacto en salud de políticas públicas dirigidas a la prevención de la obesidad ${ }^{26}$. 
Agradecimientos: Agradecemos a Laura Prieto por su valiosa colaboración en la recopilación de información y preparación de Tablas.

\section{Referencias}

1. World Health Organization, International Agency for Research on Cancer. World Cancer report 2008. Lyon; 2008.

2. Departamento de Estadísticas e Información en Salud, Ministerio de Salud, Gobierno de Chile. Estadísticas vitales. [cited 2010; Available from: http://deis.minsal. cl/deis/vitales/vita.asp].

3. Ferlay J SH, Bray F, Forman D, Mathers C and Parkin DM. GLOBOCAN 2008 v1.2, Cancer Incidence and Mortality Worldwide: IARC Cancer Base $\mathrm{N}^{\circ} 10$ [Internet]. 2010 [cited; Available from: http://globocan.iarc. $\mathrm{fr} /]$.

4. Doll R. The causes of cancer. Rev Epidemiol Sante Publique 2001; 49 (2): 193-200.

5. Rothman K, Greenland S. Modern Epidemiology: Lippincott-Raven, 1998.

6. Moreno-Altamirano A, López-Moreno S, Corcho-Berdugo A. [Main measurements in epidemiology]. Salud Pública Mex 2000; 42 (4): 337-48.

7. Peto R. The fraction of cancer attributable to lifestyle and environmental factors in the UK in 2010. Br J Cancer; 105 Suppl 2:S1.

8. Almaskut A, Farrella P, Krewski D. Statistical methods for estimating the environmental burden of disease in Canada, with applications to mortality from fine particulate matter. Environmetrics 2012; 23: 329-44.

9. World Cancer Research Fund, American Institute for Cancer Research. Food, Nutrition, Physical Activity, and The Prevention of Cancer: A Global Perspective. Washington DC AICR; 2007.

10. Ministerio de Salud de Chile, Gobierno de Chile. Encuesta Nacional de Salud Chile. 2009-2010; 2010.

11. Organización Mundial de la Salud. Estadísticas sanitarias mundiales 2012; 2012.

12. Vio F, Albala C, Kain J. Nutrition transition in Chile revisited: mid-term evaluation of obesity goals for the period 2000-2010. Public Health Nutr 2008; 11 (4): 40512.

13. Garmendia ML, Corvalán C, Uauy R. Addressing malnutrition while avoiding obesity: minding the balance. European Journal of Clinical Nutrition doi:10.1038/ ejcn.2012.190

14. Flegal KM, Graubard BI, Williamson DF. Methods of calculating deaths attributable to obesity. Am J Epide- miol 2004; 160 (4): 331-8.

15. Ministerio de Salud de Chile. Subsecretaría de Salud Pública Gobierno de Chile. Informe final Estudio de carga de enfermedad y carga atribuible, Chile. 2007 [cited; Available from: http://epi.minsal.cl/epi/html/frames/ frame2.htm].

16. Flegal KM, Williamson DF, Pamuk ER, Rosenberg HM. Estimating deaths attributable to obesity in the United States. Am J Public Health 2004; 94 (9): 1486-9.

17. Cheraghi Z, Poorolajal J, Hashem T, Esmailnasab N, Doosti Irani A. Effect of body mass index on breast cancer during premenopausal and postmenopausal periods: a meta-analysis. PLoS One 2012; 7 (12): e51446.

18. Etches V, Frank J, Di Ruggiero E, Manuel D. Measuring population health: a review of indicators. Annu Rev Public Health 2006; 27: 29-55.

19. Gobierno de Chile Ministerio de salud. Evolución de la situación nutricional en Chile, 1994-2006. Santiago, Chile; 2007.

20. World Cancer Research Fund/American Institute for Cancer Research. Policy and Action for Cancer Prevention. Washington DC: AICR; 2009.

21. Mozaffarian D, Afshin A, Benowitz NL, Bittner V, Daniels SR, Franch HA, et al. Population approaches to improve diet, physical activity, and smoking habits: a scientific statement from the American Heart Association. Circulation 2012; 126 (12): 1514-63.

22. Kahn EB, Ramsey LT, Brownson RC, Heath GW, Howze $\mathrm{EH}$, Powell KE, et al. The effectiveness of interventions to increase physical activity. A systematic review. Am J Prev Med 2002; 22 (4 Suppl): 73-107.

23. Cavill N, Bauman A. Changing the way people think about health-enhancing physical activity: do mass media campaigns have a role? J Sports Sci 2004; 22 (8): 771-90.

24. Ogilvie D, Egan M, Hamilton V, Petticrew M. Promoting walking and cycling as an alternative to using cars: systematic review. BMJ 2004; 329 (7469): 763.

25. deNazelle A, Nieuwenhuijsen MJ, Anto JM, Brauer M, Briggs D, Braun-Fahrlander C, et al. Improving health through policies that promote active travel: a review of evidence to support integrated health impact assessment. Environ Int; 37 (4): 766-77.

26. World Health Organization. The world health report Reducing Risks, Promoting Healthy Life; 2002.

27. Engeland A, Tretli S, Austad G, Bjorge T. Height and body mass index in relation to colorectal and gallbladder cancer in two million Norwegian men and women. Cancer Causes \& Control 2005; 16 (8): 987-96.

28. Silvera SA, Rohan TE, Jain M, Terry PD, Howe GR, Miller AB. Glycaemic index, glycaemic load and risk of endometrial cancer: a prospective cohort study. Public 
Obesidad y cáncer en Chile: estimación de las fracciones atribuibles poblacionales - M. L. Garmendia et al

Health Nutr 2005; 8 (7): 912-9.

29. Lindblad M, Rodríguez LA, Lagergren J. Body mass, tobacco and alcohol and risk of esophageal, gastric cardia, and gastric non-cardia adenocarcinoma among men and women in a nested case-control study. Cancer Causes Control 2005; 16 (3): 285-94.

30. van den Brandt PA, Spiegelman D, Yaun SS, Adami HO, Beeson L, Folsom AR, et al. Pooled analysis of prospective cohort studies on height, weight, and breast cancer risk. Am J Epidemiol 2000; 152 (6): 514-27.

31. Michaud DS, Giovannucci E, Willett WC, Colditz GA, Stampfer MJ, Fuchs CS. Physical activity, obesity, height, and the risk of pancreatic cancer. Jama-Journal of the American Medical Association 2001; 286 (8): 921-9.

32. Bjorge T, Tretli S, Engeland A. Relation of height and body mass index to renal cell carcinoma in two million Norwegian men and women. Am J Epidemiol 2004; 160 (12): 1168-76. 